\title{
Claude Simon, Le Cheval (1958), Les éditions du Chemin de fer, 2015, $91 \mathrm{p}$.
}

Dominique Viart

\section{OpenEdition}

1 Journals

Édition électronique

URL : http://journals.openedition.org/ccs/391

DOI : $10.4000 /$ ccs.391

ISSN : 2558-782X

Éditeur :

Presses universitaires de Rennes, Association des lecteurs de Claude Simon

\section{Édition imprimée}

Date de publication : 8 juillet 2016

Pagination : 267-271

ISBN : 978-2-7535-4876-3

ISSN : 1774-9425

Référence électronique

Dominique Viart, « Claude Simon, Le Cheval (1958), Les éditions du Chemin de fer, 2015, 91 p. », Cahiers Claude Simon [En ligne], 11 | 2016, mis en ligne le 15 septembre 2017, consulté le 24 septembre 2020. URL : http://journals.openedition.org/ccs/391 ; DOI : https://doi.org/10.4000/ccs.391 
Claude Simon, Le Cheval (1958), Les éditions du Chemin de fer, 2015, 91 p.

Désolé de n’avoir pas été véritablement l'éditeur de Claude Simon, Maurice Nadeau rappelait volontiers avoir cependant "publié la première ébauche [de La Route des Flandres] dans [sa] revue". Il est vrai que Les Lettres nouvelles et leur directeur se sont montrés très attentifs à l'œuvre naissante de l'écrivain et lui sont demeurés fidèles. Dès 1955, la revue avait accueilli un texte intitulé Babel ( $n^{\circ} 31$, oct. 1955), que les Cahiers Claude Simon ont republié dans leur n ${ }^{\circ}$. En 1958, elle donne, en deux livraisons (février et mars) Le Cheval, que les éditions du Chemin de Fer viennent de rééditer. Au cours des années suivantes, la revue mettra encore à son sommaire quatre contributions: non seulement Matériaux de construction (déc. 1960, p. 112-122), Inventaire (fév.1962, p. 50-58) et Des roches striées vert pâle parsemées de points noirs (juin-août 1964, p. 53-68), mais aussi Mot à mot (avr.1959, p. 6-10) qu'oublie étonnamment la postface de la présente réédition du Cheval (note 10, p. 79) bien que les Cahiers Simon aient également redonné ce texte dans leur $\mathrm{n}^{\circ} 5$. Les Lettres nouvelles offrent en outre régulièrement des recensions des romans de Claude Simon, notamment sous la plume d'Olivier de Magny (Le Vent en 1957, puis L'Herbe en décembre 1958). Mais la revue cesse de paraître en 1977 après avoir été relancée, et il est désormais difficile de se procurer les fascicules parus.

Aussi faut-il se réjouir de la réédition du Cheval en un volume isolé et de l'effet que produit cette publication sous forme de livre. Ce texte, paru deux ans avant La Route des Flandres a souvent été perçu par la critique comme esquisse du roman à venir. Il est vrai qu'il en dessine certains linéaments et développe plusieurs thèmes essentiels que La Route retravaillera. Mais il serait réducteur de n'y voir qu'un "avant-texte", car Le Cheval a déjà tout d'un travail achevé - du moins si l'on accepte l'idée d'un "achèvement " proprement simonien, qui ne clôt pas l'œuvre sur elle-même mais laisse les choses dans le suspens qui les caractérise. Car à la différence d'une nouvelle traditionnelle, Le Cheval ne se "conclut " pas, même si le texte se déploie comme une boucle avec in fine le retour de ses motifs initiaux: noirceur de la nuit dans laquelle les cavaliers sont immergés, et ces fleuves que l'on passe, que l'on croit ou espère passer, de la Meuse à l'Elbe, parce que leur franchissement signerait la fin de la guerre. Le récit est en effet un instantané de la Seconde Guerre mondiale, comme une latence, avant que celle-ci ne manifeste la violence à laquelle les hommes se savent destinés. C'est la saisie d'un fragment de temps, avec tout ce qui s'y entremêle, s'y heurte, mais aussi s'en émancipe, dans les conversations des soldats et les méditations du narrateur. Un régiment de cavaliers - des "dragons »- avance dans la nuit, sous la pluie, au cœur de cette « drôle de guerre » 
qui n'allait pas tarder à s'affirmer meurtrière (ce que non seulement le texte pressent, mais qu'il évoque aussi par anticipation), fait halte dans un village, tout au plus un hameau, y demeure une journée, le temps de saisir les linéaments un peu flous d'une histoire d'adultère rural, de cantonner et de repartir avant le lever du soleil.

Entièrement narré à la première personne, et non dans l'hésitation énonciative caractéristique de La Route des Flandres, le texte rassemble ainsi plusieurs motifs insistants: aux dialogues marqués de gouaille et d'ironie entre le narrateur, son ami Maurice (c'est là le véritable prénom d'un compagnon du brigadier Simon, qui deviendra Blum dans La Route des Flandres, mais sans qu'il soit besoin de le souligner par son patronyme, le personnage est déjà explicitement juif dans ce court récit, ce qui l'expose à un antisémitisme ordinaire qu'il moque avec causticité) et deux autres soldats, se mêlent discussions et réflexions au sujet d'un cheval malade, blessé par les coups sur la tête que lui a infligés son cavalier, et dont l'agonie donne lieu à une méditation sur la mort. À cela se conjuguent des réflexions, issues de réminiscences perceptives insistantes - la chevauchée au pas dans la nuit noire, la chair laiteuse d'une femme entr'aperçue - d'autres réflexions qui évoquent la situation de ces hommes bientôt sacrifiés, livrés au néant à venir, et entraînent les pensées du narrateur dans un hors temps visionnaire, véritable synthèse de l'Histoire et des guerres qui la scandent.

Si le récit s'ouvre sur le début de la nuit, peu avant la halte, et se clôt sur une aube non encore advenue (" quatre heures du matin »), le temps y est comme suspendu, nié: "Le temps n'existe pas. Ni demain, ni hier. "Quarante-huit heures, dit le texte, sans que l'on sache quand elles ont eu lieu. Quarante-huit heures qu'il serait hasardeux de comparer à un temps tragique, tant le temps est ici désaccordé, incertain, distendu entre les scènes et dialogues rapportés, et les méditations qui le diffractent, avalé par cette phénoménologie perceptive d'où s'engendre un flux d'images archaïques et hallucinées. Tout Simon est déjà là, dans le double régime de son écriture, attentive, caustique et métaphorique à la fois, riche d'une puissance analogique qui s'empare du sens et des sensations.

Déjà là, mais pas tout à fait cependant. C'est en quoi, au-delà de sa réussite effective comme tel, $\mathrm{Le}$ Cheval est aussi un texte précieux dans l'avènement de l'œuvre à venir. Car tout autonome qu'il soit indiscutablement, il appartient à la galaxie de La Route des Flandres, roman durant vingt ans cherché, repris et retravaillé avant que d'aboutir, qui y puise non seulement des scènes et des thèmes, mais aussi des formules et des phrases entières. Roman dont on rappellera que, parmi les titres possibles, essayés sur plusieurs pages de manuscrit, figurait justement "Les chevaux ", comme une expansion plurielle de ce texte initial, venant adjoindre d'autres montures au "cheval " ici agonisant: celui sur lequel de Reixach trouvera la mort, ceux de la course hippique où se joue un autre conflit adultérin et celui avec lequel Georges essaie d'échapper aux tirs ennemis. Aussi est-ce également dans cette perspective que cette réédition nous importe et nous éclaire, comme premier essai de 
" mise en ordre » de fragments - scènes et dialogues - dont le roman sera lui-même, quelques mois plus tard, constitué.

Dès lors, pour un familier de l'œuvre de Simon et tout particulièrement de La Route des Flandres, apparaît au regard de ce roman tout un travail sur le genre littéraire et sur la phrase.

Sur le genre littéraire d'abord, le passage du texte bref au roman est en effet très instructif. Contrairement à ce que l'on pourrait imaginer, il ne se fait pas - du moins pas simplement - par adjonctions et développements, mais aussi par retranchements. Car si bien des motifs viennent s'agréger dans La Route à ceux disposés dans Le Cheval (l'épisode central de la mort du Capitaine, l'évocation de Corinne, de sa liaison avec Iglésia, les mentions du père du narrateur et de l'ancêtre...), ceux présents ici subissent en revanche de forts allègements: disparaissent une grande partie des dialogues, notamment ceux avec les paysans du cantonnement et avec certains soldats, certains personnages (Leclerc, plusieurs interlocuteurs du village), ainsi que l'essentiel de l'enquête autour de l'adultère paysan. Soit plus précisément: le pittoresque et le romanesque. Le roman simonien sera donc cela: un roman qui ne fait allégeance à aucun de ces deux régimes d'écriture, mais au contraire s'en dépossède vivement, pour n'en plus conserver que l'esquisse, la trace allusive.

Au sujet de la phrase ensuite, car bien des formules connues résonnent ici dans l'oreille du lecteur. Les phrases du Cheval éclairent souterrainement celles de La Route des Flandres qui en reprennent les mouvements et les formules. Ainsi, et de manière si frappante, ce segment: "les hommes qui ne font que naître et mourir, passer " (p. 13) donne son rythme à l'incipit du roman: " je pouvais voir aller et venir passer les taches rouges acajou ocre des chevaux qu'on menait à l'abreuvoir " (EI, p. 197). Et le lecteur de comprendre tout à coup combien cet « aller et venir passer ", qui lui semblait à la première lecture résonner d'un autre sens que celui de simples chevaux allant et venant, se trouve effectivement déjà lesté d'une réflexion latente sur la condition humaine.

Plus généralement, la confrontation de passages semblables d'une œuvre à l'autre fait apparaître une densification de l'écriture romanesque. Un exemple le montre à l'évidence. Il s'agit de cette femme apparue, sur laquelle revient le narrateur:

et tandis que je dessellais il me semblait toujours la voir, là où elle s'était tenue l'instant d'avant, ou plutôt la sentir, la percevoir - car je l'avais si peu, si mal vue - plus avec mes autres sens, plus avec tout le restant de mon corps que par la fugitive image qu'elle avait laissée sur ma rétine et qu'il m'était impossible de reconstituer alors que je pouvais non la voir, mais pour ainsi dire la connaître: une chose tiède, étrangement nue, laiteuse comme le lait qu'elle venait de tirer au moment où nous étions entrés, une sorte d'apparition non pas éclairée par la lanterne mais bien plutôt lumineuse par elle-même, comme si sa peau diaphane était elle-même la source de cette lumière, comme si toute cette interminable chevauchée nocturne à l'assaut des montagnes n'avait eu d'autre raison, d'autre but que la découverte, au bout des ténèbres, de cette argile blanche modelée au sein de la nuit, semblable à une de ces figurines d'envoûtement: non une femme, mais l'idée même, le symbole de toute femme et de toute paix, tel que, dans notre célibat forcé, notre 
jeunesse frustrée, affamée, nous pouvions le concevoir, c'est-à-dire [...], sommairement façonnée avec le pouce dans la tendre pâte, la douce chair de femme, deux cuisses, un ventre, deux seins, la ronde colonne du cou et au creux de tout cela, comme au centre de ces statues nègres à la précise, tranquille et glorieuse impudeur, l'antre humide et noir, cette bouche herbue aux âcres senteurs de terre, d'humus, de coquillage, semblable à une source sous les broussailles, sable humide aux lèvres altérées du voyageur, du pèlerin, du soldat perdu, abandonné aux effrayantes ténèbres de la nuit et de la mort: le doux, l'apaisant refuge, le sein profond de l'oubli. (p. 15-16)

De ce long passage, précis et très explicatif, La Route des Flandres ne retient que quelques bribes, que le roman en outre disperse. Soit ici le fragment principal:

il lui semblait toujours la voir, là où elle s'était tenue l'instant d'avant, ou plutôt la sentir, la percevoir comme une sorte d'empreinte persistante, irréelle, laissée moins sur sa rétine (il l'avait si peu, si mal vue) que, pour ainsi dire, en lui-même: une chose tiède, blanche comme le lait qu'elle venait de tirer au moment où ils étaient arrivés, une sorte d'apparition non pas éclairée par cette lampe mais luminescente, comme si sa peau était elle-même la source de la lumière, comme si toute cette interminable chevauchée nocturne n'avait eu d'autre raison, d'autre but que la découverte à la fin de cette chair diaphane modelée dans l'épaisseur de la nuit: non pas une femme mais l'idée même, le symbole de toute femme, c'est-à-dire [...] sommairement façonnés dans la tendre argile deux cuisses un ventre deux seins la ronde colonne du cou et au creux des replis comme au centre de ces statues primitives et précises cette bouche herbue cette chose au nom de bête, de terme d'histoire naturelle - moule poulpe pulpe vulve - faisant penser à ces organismes marins et carnivores aveugles mais pourvus de lèvres, de cils: l'orifice de cette matrice le creuset originel qu'il lui semblait voir dans les entrailles du monde, semblable à ces moules dans lesquels enfant il avait appris à estamper soldats et cavaliers. (EI, p. 219-220)

Le lecteur constatera que toutes les notations psychologiques, toute allusion au " célibat forcé ", à l'aspiration au réconfort du soldat, à l'impudeur de l'art nègre, ont disparu, ainsi que le lyrisme pathétique du "soldat perdu, abandonné aux effrayantes ténèbres de la nuit et de la mort ", bref tout ce qui relève du topos et du commentaire. Le texte du roman se donne ainsi plus de concentration et d'ampleur, avec notamment plus d'ouverture à l'archaïque (statue primitive vs art nègre, organismes marins...) d'une part, au jeu érotique des signifiants d'autre part (moule, poulpe, pulpe, vulve). Moins explicite, le texte devient plus efficace.

Ce qui émerge ainsi, c'est tout le phrasé de Simon, sa déprise et sa densification progressives, sa profusion analogique, ses collusions lexicales. Tout le contraire de ce néo-classicisme, dont l'affuble non sans grandiloquence la postface de cette réédition pour qui le texte du Cheval « apparaît tel qu'en lui-même œuvré: un pur cristal taillé, facetté avec art » (sic). Cette complaisance rhétorique de la présentation éditoriale serait vénielle, si elle ne faussait considérablement l'esthétique simonienne en tirant l'œuvre vers je ne sais quelle poétique de la "pureté ». Ni styliste, ni ciseleur, Simon accueille au contraire les images qui lui viennent, donne de l'empan à la phrase, laisse libre cours à ses dérives, non sans en densifier les formulations. S’il 
fallait - mais est-ce bien nécessaire? - l'inscrire dans quelque parenté esthétique, c'est plutôt vers le baroque qu'il conviendrait de se tourner: un baroque auquel Simon fait lui-même maintes allusions et dont il a photographié plus d'une fois les réalisations. Barroco: cette pierre irrégulière (barroca), comme une "moraine ", emblématise un art du divergent, de l'impur, qui mêle, comme ici, le trivial et le sublime, et tient sa puissance de leur friction. Car c'est bien cela, Le Cheval, un texte qui, pour dire la guerre, atteint à une hauteur nouvelle, s'émancipe peu à peu des lignes attendues et circonscrites de la forme romanesque, n'en retient que quelques bribes esquissées, livrées au sarcasme, et comme emportées dans un monde en dérive, "pris dans cette chose inhumaine, monstrueuse, sans mesure, qui rabotait sous son poids effrayant la surface du monde ([...] : cette olympienne, froide, et indifférente progression de l'Histoire, ce lent glacier en marche depuis les temps immémoriaux, broyant, écrasant tout, avançant sans trêve entre les moraines rejetées de nos ossements) » (p. 52). Non, rien vraiment de la préciosité cristalline à laquelle on le voudrait indexer, mais l'irrépressible flot qui détruit sur son passage les vies, les hommes et les chevaux.

\section{Dominique Viart}

Gastone Novelli, Voyage en Grèce, Lyon, éditions Trente-trois morceaux, 2015, 105 p., 16 ill. couleurs.

L'ouvrage de Gastone Novelli (1925-1968) que viennent de publier à Lyon les éditions Trente-trois morceaux sous le double titre Voyage en Grèce Viaggio in Grecia rassemble, en un élégant petit volume, plusieurs documents peu accessibles et qui s'éclairent mutuellement.

Il permet tout d'abord de découvrir le texte publié en 1966 à Rome par les éditions Arco d'Alibert. Jusqu'alors inédit en français, il est présenté ici en version bilingue avec la traduction de Thierry Gillybœuf. Le titre, les indications de dates, les noms de lieux pourraient inciter à le lire comme le carnet d'un périple accompli par le peintre dans le Péloponnèse et les îles grecques, jusqu'en Crète et à Rhodes, entre 1961 et 1963. La dernière phrase « Aujourd'hui, à dix heures du matin de la fin août 1963, deux ans jour pour jour après avoir commencé, le voyage est terminé " confirmerait cette lecture si celle-ci n'avait commencé sur un mode nettement moins référentiel: "il existe un territoire, assez grand, couvert de montagnes, inconnu de tous, qui n'est signalé sur aucune carte». De fait, le texte évoque les souvenirs de nombreux voyages en Grèce que Novelli a effectués au cours de cette période, mais aussi des expériences inoubliables faites au Brésil où il a séjourné dans les années 1950, et propose un itinéraire sensoriel, érotique, imaginaire et poétique: "Le seul et unique guide qui vaille pour ce voyage, ce sont nos sens. »

Aux six gravures de l'édition originale, les éditions Trente-trois morceaux, qui portent « un intérêt particulier [...] aux frontières poreuses entre les médiums, par 